\title{
ASSESSING THE INFLUENCE OF INCREASED STRESS ON BRUXISM DURING THE COVID-19 PANDEMIC
}

\author{
Dimitar Kirov $^{1}$, Lilia Doichinova ${ }^{2}$, Elitsa Deliverska ${ }^{3}$, Janet Kirilova ${ }^{4}$ \\ 1) Department of Prosthetic Dental Medicine, Faculty of Dental Medicine, Medi- \\ cal University, Sofia, Bulgaria \\ 2) Department of Pediatric Dental Medicine, Faculty of Dental Medicine, Medi- \\ cal University, Sofia, Bulgaria \\ 3) Department of Oral and Maxillofacial Surgery Faculty of Dental Medicine, \\ Medical University, Sofia, Bulgaria \\ 4) Department of Conservative Dentistry, Faculty of Dental Medicine, Medical \\ University, Sofia, Bulgaria.
}

\section{SUMMARY:}

Aim of the study: This study aims to evaluate the possible relationship between the occurrence of bruxism and perceived stress during the Covid-19 pandemic period.

Material and methods: Two questionnaires were administered to one hundred forty-five (145) respondents randomly selected from different age groups and gender. Each patient was screened with and Perceived Stress Scale-10 (PSS-10) containing ten questions. The questionnaire assessed patients about stress related to everyday problems, personal events, and coping methods. The answers were scored using an ordinal five-point scale. In this study, a sixitem self-administered questionnaire was used to evaluate self-reported awake and sleep bruxism.

Results: In the present study, bruxism was not statistically significantly correlated with self-reported perceived stress based on PSS-10 (rank correlation coefficient was rho $(145)=0.080, p<0.341)$. There was no statistically significant difference within the study group between genders in the frequency of low, moderate, and high PSS-10 results (U $=2329.5, p=0.339$ ). The analysis also showed no statistically significant difference between males and females regarding bruxism score $(\mathrm{U}=2381.5, \mathrm{p}=0.434)$.

Conclusions: Results do not confirm the findings of a positive association between bruxism and perceived stress. Within the limitations of this study, the data suggest that the relationship between perceived stress and the expression of bruxism is more complex. Given the results of the current research, this issue requires further investigation.

Keywords: bruxism, coronavirus pandemic, COVID19, perceived stress,

\section{INTRODUCTION}

Bruxism is repetitive muscle activity characterized by clenching or grinding of the teeth or bracing or thrusting the mandible and specified as either sleep bruxism or awake bruxism [1].
Theories about the origin of bruxism have hypothesized different factors: peripheral, connected to teeth occlusion interference, central, connected to neurotransmission from brain to chewing muscles, and psycho-social, associated with stress [2, 3].

The appearance of bruxism can be seen in the population at each age, starting from the eruption of deciduous teeth [4]. Recently, the number of patients suffering from bruxism has increased significantly. The incidence of bruxism has been reported to vary between $8 \%$ and $31 \%$ of the population and can lead to headaches and pain in the teeth, muscles, and jaw [5, 6].

Situations like the COVID-19 pandemic can lead to psychological issues and high-stress levels. Drastically altered was routine life, and Quarantine and self isolation can hurt people's physical and mental health [7].

The common psychological responses of the people to the Coronavirus pandemic included stress, anxiety, and depression [8]. All these can influence oral health and trigger various conditions, such as bruxism, sleep disorders, and TMD [9].

The etiology of bruxism is multi-factorial. The factors associated with bruxism development are smoking, high alcohol and coffee consumption, anxiety, and depression [10]. Recent studies show that emotional changes may be associated with bruxism $[11,12]$. It is generally accepted that stressful situations can lead to occlusal parafunction and temporomandibular disorders without being the only cause [13].

Several studies reported that bruxism, one of the most common parafunctional habits, has psycho-social and emotional components as a contributing factor [14, 15].

The harmful habit causes changes in the structure of the masticatory system, causing teeth to wear, inflammation, muscle pain, and cracking sounds in the joint. Psycho-social factors such as anxiety and perceived stress are as important as somatic causes in the occurrence and maintenance of bruxism $[16,17]$. 
Some studies do not support the positive relationship between bruxism and perceived stress [18, 19].

This study evaluates the possible relationship between bruxism and perceived stress during the Covid-19 pandemic.

\section{MATERIAL AND METHODS}

Two questionnaires were administered to one hundred forty-five (145) respondents randomly selected from different age groups and gender. Data obtained from questionnaires revealed 85 female participants $(58.6 \%)$ and 60 male participants $(41.4 \%)$. Age varied widely among respondents, from 27 to 61 years of age (Mean 48.33 \pm 0.55 ; $\mathrm{SD}=6.68$ ).

The quantity of the stress is evaluated with the Perceived Stress Scale-10 (PSS-10). The PSS-10 measured selfreported stress and is used for its excellent validity and reliability. It includes ten questions, with answers ranked by a 5-point Likert scale, and assesses stressful experiences and responses to stress over the previous four weeks. PSS-10 scores are obtained by reversing the four positive items, $0=4,1=3,2=2,3=2,4=1$, and then summing across all ten items. The final result is the sum of 10 individual questions. The maximum number of points obtained for each participant is 40 . According to the PSS-10, scores ranging from 0 13 were considered low perceived stress, from 14-19 were deemed moderate perceived stress, and 20-40 were supposed to be high perceived stress.

Bruxism was investigated using a six-item self-report questionnaire as follow:1) Do you grind or clench your teeth during sleep?; 2) Do you notice to grind or clench your teeth when you are awake?; 3) Do you feel soreness or fatigue of the jaw when you wake up?; 4) Does the temple area of your headaches early in the morning?; 5) Do you have any worn-down dentition?; 6) Do you have any limitations opening the mouth? The answers were scored using an ordinal five-point scale including 0 (never), 1 (sometimes), 2 (regularly), 3 (often), and 4 (always). Each question was formulated as follows: "for each of the following conditions, please indicate how often you have recently engaged in them?"'The answers were coded using an ordinal five-point scale, including 0 (never), 1 (sometimes), 2 (regularly), 3 (often), and 4 (always). The patients were instructed as follows: "for each of the following conditions, please note how often you have had it in the last year?".

Respondents were provided with detailed information on the study protocol, and informed consent was obtained before the administration of the questionnaires.

\section{Statistical Analysis}

For qualitative variables, the structure indices were calculated and expressed in \%. Data were summarized by calculating a descriptive statistical index. As the distributions of variables significantly differed from the normal distribution, the non-parametric Mann-Whitney $\mathrm{U}$ test is usually used to check differences between genders. The chisquare test was used for two categorical variables. The relationship between variables was analyzed with Spearman's rank correlation coefficient. A level of $\mathrm{p}<0.05$ was considered statistically significant. SPSS software (version 20; IBM, Tokyo, Japan) was used for all statistical analyses.

\section{RESULTS}

In this study, a total of 145 participants were evaluated aged 27 to 61 years, $58.6 \%$ of women (mean age 47.6 \pm 6.98 ) and $41.4 \%$ of men (mean age $48.8 \pm 6.47$ years) filled out the questionnaires (Table 1).

Table 1. Descriptive statistics of the study population

\begin{tabular}{|c|c|c|c|}
\hline \multirow{2}{*}{ Gender } & \multicolumn{3}{|c|}{$\begin{array}{c}\text { Distribution of the study } \\
\text { population }\end{array}$} \\
\cline { 2 - 4 } & $\mathrm{n}$ & $\%$ & Mean age $( \pm \mathrm{SD})$ \\
\hline Male & 60 & 41.4 & $48.8(6.47)$ \\
\hline Female & 85 & 58.6 & $47.6(6.98)$ \\
\hline Total & 145 & 100 & $48.3(6.69)$ \\
\hline
\end{tabular}

The results obtained in this work indicated increased levels of stress level among the studied population. The mean results in PSS 10 stated a level of perceived stress in the study group was 19.44 points $(6.0 \div 36.0)$. A high level of anxiety was observed in $35(24.1 \%)$ of the respondents, a moderate level in $68(46.9 \%)$, and a low level in $42(29 \%)$. Most respondents were considered to perceive medium and high-stress levels $(71 \%)$ based on PSS 10 results (Table 2 ).

Table 2. Distribution of perceived stress and bruxism score

\begin{tabular}{llcc}
\hline & & $\mathrm{n}$ & $(\%)$ \\
\cline { 3 - 4 } Exposure & Low perceived stress & 42 & 29 \\
to stress & Moderate perceived stress & 68 & 46,9 \\
& Highperceived stress & 35 & 24,1 \\
\hline \multirow{4}{*}{ Bruxism } & Never & 91 & 62,8 \\
presents & Sometimes & 10 & 6,89 \\
& Regularly & 17 & 11,7 \\
& Often & 21 & 14,5 \\
& Always & 6 & 4,13 \\
\hline
\end{tabular}

Mann-Whitney U test is usually used to check whether there are differences between genders. The analysis also showed no statistically significant difference between genders in terms of bruxism score $(\mathrm{U}=2381.5, \mathrm{z}=$ $.782, p=0.434$ ) (Table 3 ). In the present study, there was no statistically significant difference within the study group between genders in the frequency of low, moderate, and high PSS-10 results $(\mathrm{U}=2329.5, \mathrm{z}=-.955, p=0.339)$.

Table 3. Differences between perceived stress and bruxism score according to gender

\begin{tabular}{lccc} 
& \multicolumn{3}{c}{ Gender } \\
\cline { 2 - 4 } & $\mathrm{U}$ & $\mathrm{z}$ & $\mathrm{p}$ \\
\cline { 2 - 4 } Bruxism score & 2381.5 & -.782 & 0.434 \\
Perceived stress & 2329.5 & -.955 & 0.339 \\
\hline
\end{tabular}


The Spearman rho rank correlation coefficient was used to establish the correlation between bruxism and levels of perceived stress in the studied population (Table 4).

Table 4. Spearman correlations for bruxism and perceived stress

\begin{tabular}{|c|c|c|c|}
\hline \multicolumn{4}{|l|}{ Perceived stress } \\
\hline \multirow{3}{*}{ Spearman's rho } & \multirow{3}{*}{ Bruxism } & $\begin{array}{l}\text { Correlation } \\
\text { coefficient }\end{array}$ & $0.080^{*}$ \\
\hline & & Sig. (2-tailed) & 0.341 \\
\hline & & $\mathrm{N}$ & 145 \\
\hline
\end{tabular}

* There is no correlation at the 0.05 level (2-tailed).

In the study population, a positive correlation between the PSS-10 score and the bruxism was not found (rank correlation coefficient was rho $(145)=0.080, p<$ $0.341)$.

\section{DISCUSSION}

The studies on the relationship between bruxism and risk factors as stress have increased in recent years. However, the relationship between the occurrence of bruxism and perceived stress is still controversial because it has not been scientifically proven that stress can be the cause of the occurrence and aggravation of bruxism. The presented study investigated the relationship between bruxism and psycho-emotional condition assessed based on the Perceived Stress Scale (PSS-10) results.

Many scientific reports indicate a possible connection between bruxism and stress. Saczuk K et al., in the study on self-reported bruxism and perceived stress, reported that participants reporting sleep bruxism showed higher scores of PSS-10 [20]. The psycho-emotional component is indicated as an important etiological factor for bruxism.

There are scientific studies that only partially support the relationship between bruxism and perceived stress. In a research of the prevalence of awake and sleep bruxism and its correlation with stress, Cavallo et al. reported that correlation between stress and bruxism exists only for the male gender [2]. Other authors concluded that diurnal bruxism seems to be associated with psycho-social factors, whereas there was no evidence to relate sleep bruxism with psychological stress [21]. The results described in the present study are consistent with several earlier investigations $[12,22]$. The current study results do not support evidence from other researches that bruxism is positively correlated with psychological stress.

Ohlmann et al., in the study, aimed to identify associations between definite sleep bruxism and chronic stress and sleep quality, reported that chronic stress and sleep quality do not seem to be associated with sleep bruxism
[12]. These results are consistent with our findings as well. Contrary to the common opinion, some authors hypothesize that bruxism is not always correlated with increased stress. Smardz J et al. declared that intensity of sleep bruxism was not statistically significantly associated with self-reported perceived stress [18]. The results are consistent with our research.

In contrast to our results, some studies prove that stress causes an increase in the muscular activity of the masticatory system, which in turn increases and prolongs the duration of dental contacts [26]. Demjaha $G$ et al. have shown that the prevalence of bruxism is growing related to stress, changes in lifestyle, lousy nutrition, and sleep problems [4].

In a systematic review, Chemelo VDS et al. gathered evidence of the association between stress and bruxism, confirmed in the quantitative analysis [13]. Contrary to our findings, Quadri MF et al. reported that patients with highstress levels are almost six times more likely to report awake bruxism [23]. In public mental health terms, the significant psychological impact to date is elevated rates of stress or anxiety. Researches have demonstrated that the Coronavirus pandemic has caused significant adverse effects on the psycho-emotional status of populations, resulting in the intensification of bruxism and TMD symptoms [7]. According to these studies, anxiety, depression, and the stress evoked by the Coronavirus pandemic increased the prevalence of TMD and bruxism [24, 25].

However, advances in research point to the more significant impact of somatic rather than psychological factors. Thus, the current study's findings support the idea that stress is not an initiating factor for bruxism.

Our study investigated bruxism and perceived stress based on self-report only. The main limitation of this study was that the self-report questionnaire with closed questions hampered an in-depth exploration of the participants' subjective state. It remains limited to the ability to express themselves and their feelings.

\section{CONCLUSIONS}

Within the limitations of the present study, it can be concluded that there is no relationship between perceived stress and bruxism. Results do not confirm the findings of the positive association between bruxism and perceived stress. High neuromuscular tension in the masticatory system can be the result of stress, but we should bear in mind that the stress response is largely dependent on personality and other predisposing factors. In conclusion, it could be suggested that personality characteristics are more important factors in the pathogenesis of bruxism than the stress itself. These findings suggest that additional studies are warranted to clarify the link between bruxism and psychological stress. The subject of the influence of the psycho-emotional state on bruxism should be further explored. 


\section{REFERENCES:}

1. Lobbezoo F, Ahlberg J, Raphael KG, Wetselaar P, Glaros AG, Kato $\mathrm{T}$, et al. International consensus on the assessment of bruxism: Report of a work in progress. J Oral Rehabil. 2018 Nov;45(11):837-844. [PubMed]

2. Cavallo P, Carpinelli L, Savarese G. Perceived stress and bruxism in university students. BMC Res Notes. 2016 Dec 21;9(1):514. [PubMed]

3. Flueras MI, Bocsan IC, Buduru S, Pop RM, Vesa SC, Zaharia A, et al. The correlation between sleep bruxism, salivary cortisol, and psychological status in young, Caucasian healthy adults. Cranio. 2019 May;39(3):218224. [PubMed]

4. Demjaha G, Kapusevska B, Pejkovska-Shahpaska B. Bruxism Unconscious Oral Habit in Everyday Life. Open Access Maced J Med Sci. 2019 Mar 14;7(5):876-881. [PubMed]

5. Manfredini D, Serra-Negra J, Carboncini F, Lobbezoo F. Current Concepts of Bruxism. Int $J$ Prosthodont. 2017 Sep-Oct;30(5): 437-438. [PubMed]

6. Manfredini D, Winocur E, Guarda-Nardini L, Paesani D, Lobbezoo F. Epidemiology of bruxism in adults: A systematic review of the literature. J Oral Facial Pain. 2013 Spring;27(2):99-110. [PubMed]

7. Emodi-Perlman A, Eli I, Smardz J, Uziel N, Wieckiewicz G, Gilon E, et al. Temporomandibular Disorders and Bruxism Outbreak as a Possible Factor of Orofacial Pain Worsening during the COVID-19 Pandemic-Concomitant Research in Two Countries. J Clin Med. 2020 Oct 12;9(10):3250. [PubMed]

8. Wang C, Pan R, Wan X, Tan Y, $\mathrm{Xu}$ L, Ho CS, et al. Immediate Psychological Responses and Associated Factors during the Initial Stage of the 2019 Coronavirus Disease (COVID19) Epidemic among the General Population in China. Int J Environ Res Public Health. 2020 Mar 6;17(5): 1729. [PubMed]

9. Almeida-Leite CM, Barbosa JS, Conti PCR. How psycho-social and economic impacts of COVID-19 pandemic can interfere on bruxism and temporomandibular disorders? J Appl Oral Sci. 2020; 28:e20200263. [PubMed]

10.Pontes LDS, Prietsch SOM. Sleep bruxism: population based study in people with 18 years or more in the city of Rio Grande, Brazil. Rev Bras Epidemiol. 2019; 22:e190038. [PubMed]

11.Kuhn M, Türp JC. Risk factors for bruxism. Swiss Dent J. 2018 Feb 12;128(2):118-124. [PubMed]

12.Ohlmann B, Bömicke W, Habibi Y, Rammelsberg P, Schmitter M. Are there associations between sleep bruxism, chronic stress, and sleep quality? J Dent. 2018 Jul;74:101-106. [PubMed]

13. Chemelo VDS, Né YGS, Frazão DR, de Souza-Rodrigues RD, Fagundes NCF, Magno MB, et al. Is There Association Between Stress and Bruxism? A Systematic Review and Meta-Analysis. Front Neurol. 2020 Dec 7;11:590779. [PubMed]

14.van Selms M, Kroon J, Tuomilehto H, Peltomaa M, Savolainen A, Manfredini D, et al. Self-reported sleep bruxism among Finnish symphony orchestra musicians: Associations with perceived sleep-related problems and psychological stress. Cranio. 2020 Nov 30:1-8. [PubMed]

15. Drumond CL, Paiva SM, VieiraAndrade RG, Ramos-Jorge J, RamosJorge ML, Provini F, et al. Do family functioning and mothers' and children's stress increase the odds of probable sleep bruxism among schoolchildren? A case control study. Clin Oral Investig. 2020 Feb;24(2):1025-1033. [PubMed]

16.Przystañska A, Jasielska A, Ziarko M, Pobudek-Radzikowska M, Maciejewska-Szaniec Z, PryliñskaCzy ¿ewska A, et al. Psychosocial Predictors of Bruxism. Biomed Res Int. 2019 Oct 13;2019:2069716. [PubMed]

17.Goldstein G, DeSantis L, Goodacre C. Bruxism: Best Evidence Consensus Statement. J Prosthodont. 2021 Apr;30(S1):91-101. [PubMed]

18.Smardz J, Martynowicz H, Wojakowska A, Michalek-Zrabkowska
M, Mazur G, Wieckiewicz M. Correlation between Sleep Bruxism, Stress, and Depression-A Polysomnographic Study. J Clin Med. 2019 Aug 29;8(9):1344. [PubMed]

19. Muzalev K, Visscher CM, Koutris M, Lobbezoo F. Long-term variability of sleep bruxism and psychological stress in patients with jawmuscle pain: Report of two longitudinal clinical cases. J Oral Rehabil. 2018 Feb;45(2):104-109. [PubMed]

20.Saczuk K, Lapinska B, Wilmont P, Pawlak L, LukomskaSzymanska M. Relationship between Sleep Bruxism, Perceived Stress, and Coping Strategies. Int J Environ Res Public Health. 2019 Sep 1;16(17): 3193. [PubMed]

21.Manfredini D, Lobbezoo F. Role of psychosocial factors in the etiology of bruxism. J Orofac Pain. 2009 Spring;23(2):153-66. [PubMed]

22. Sampaio NM, Oliveira MC, Andrade AC, Santos LB, Sampaio M, Ortega A. Relationship between stress and sleep bruxism in children and their mothers: A case control study. Sleep Sci. 2018 Jul-Aug;11(4):239244. [PubMed]

23.Quadri MF, Mahnashi A, Al Almutahhir A, Tubayqi H, HakamiA, Arishi M, et al. association of awake bruxism with khat, coffee, tobacco, and stress among Jazan University Students. Int J Dent. 2015;2015: 842096. [PubMed]

24.Polmann H, Réus JC, Massignan C, Serra-Negra JM, Dick BD, Flores-Mir C, et al. Association between sleep bruxism and stress symptoms in adults: A systematic review and meta-analysis. J Oral Rehabil. 2021 May;48(5):621-631. [PubMed]

25.Polmann H, Domingos FL, Melo G, Stuginski-Barbosa J, Guerra ENDS, Porporatti AL, et al. Association between sleep bruxism and anxiety symptoms in adults: A systematic review. J Oral Rehabil. 2019 May; 46(5):482-491. [PubMed]

26. Stocka A, Kuc J, Sierpinska T, Golebiewska M, Wieczorek A. The influence of emotional state on the masticatory muscles function in the 
group of young healthy adults. BioMed Research International. 2015;2015:174013. [PubMed]

27. Michalek-Zrabkowska M

Wieckiewicz M, Smardz J, Gac P,

Poreba R, Wojakowska A, et al. Deter- mination of Inflammatory Markers, Hormonal Disturbances, and Sleepiness Associated with Sleep Bruxism , Among Adults. Nat Sci Sleep. 2020 Nov 10;12:969-979. [PubMed]

Please cite this article as: Kirov D, Doichinova L, Deliverska E, Kirilova J. Assessing the influence of increased stress on bruxism during the COVID-19 pandemic. J of IMAB. 2022 Jan-Mar;28(1):4237-4241.

DOI: https://doi.org/10.5272/jimab.2022281.4237

Received: 24/03/2021; Published online: 15/02/2022

Address for correspondence:

Dimitar Kirov, Associate Professor, DDS, PhD

Department of Prosthetic Dentistry, Faculty of Dental Medicine, Medical University,

1, St. Georgi Sofyiski Str., 1431 Sofia, Bulgaria.

e-mail: dimiterkirov@gmail.com 